\title{
Umbolith: A Rare Case of Umbilical Lithiasis in Adult
}

\author{
M Abdur Rouf ${ }^{1}$, Baikaly Ferdous², Abdul Alim Sheikh³, M Ziaur Rahman ${ }^{4}$, \\ Salma Chowdhury ${ }^{5}$, Tanvirul Hasan ${ }^{6}$, ABM Moniruddin ${ }^{7}$
}

\begin{abstract}
A 20-year old non-diabetic non-hypertensive non-smoker male farmer presented with purulent umbilical discharge for one year. He used to live an unhygienic life. He had throbbing pain, intermittent fever preceded by chills and rigors for fifteen days. He was treated with various antibiotics, analgesics, antipyretics for a long time by local physicians. But he was not cured. The umbilical and periumbilical area were found inflamed, edematous and red with a palpable small hard infra umbilical mass. The mass was irreducible, non-pulsatile, non-compressible and not trans illuminating with no visible and no palpable impulse on coughing. Just prior to incision, a probe was introduced through the umbilicus that had not passed beyond the abdominal wall into the peritoneal cavity indicating that it was a sinus and not a fistula.The infra-umbilical smile incision was deepened and a flap was raised upwards until multiple small calculi of variable consistency were found at a depth. These were extracted. The sinus tract and the accompanying small abscess cavity were excised and sent to the laboratory for culture and sensitivity tests, histopathological examination and other relevant tests. The studies revealed Staphylococcus aureus sensitive to gentamycin and amikacin. The histopathological examination revealed acute inflammatory cells with no granuloma and no malignancy. The patient was given parenteral antibiotics as per the sensitivity test result plus metronidazole against possible anaerobic co-infection. The post-operative period was smooth and uneventful. We like to share our experience of managing this very rare disease of umbilical lithiasis.
\end{abstract}

Key words: Umbilical concretions(Calculi), sepsis, discharge, edema.

Date of received: 18.10 .2020

Date of acceptance: 20.05 .2021

DOI: https://doi.org/10.3329/kyamcj.v12i2.55446

KYAMC Journal.2021;12(02): 111-113.

\section{Introduction}

The 'umbilicus' is a clinical term for the 'nave'l or for its colloquial term 'belly button'. It is a weak point of scar tissue at the centre of anterior abdominal wall, covered by puckered skin. It marks the transmission of two umbilical arteries, one umbilical vein, the allantois and the omphalomesenteric (vitelline) duct within Wharton's jelly in fetal life. ${ }^{1}$ In adulthood, it appears as protuberant or flat or hollowed zone of variable depths in the abdominal wall marking the attachment of umbilical cord. ${ }^{2}$
Various disorders affecting the adult umbilicus include umbili-
cal hernias, umbilical sinus, umbilical fistula(through which
menstrual or fecal or urinary discharge can occur), umbilical
endometriosis, urachal cyst or sinus, vitelline cyst or sinus, hair
tufts with infection, pilonidal sinus and foreign bodies, Omphali-
tis (inflammation of umbilicus), umbilical infection [and
complications thereof like sepsis, cellulitis, portal pyemia,
portal vein thrombosis, liver abscess, septicemia, septic shock,
MODS (Multi Organ Dysfunction Syndrome), MSOF

1. Associate Professor, Department of Surgery, KhwajaYunus Ali Medical College Hospital, Sirajgonj, Bangladesh.

2. Medical Officer (MO), Shaheed M Monsur Ali Medical College, Sirajganj.

3. Assistant Professor, Department of Surgery, North Bengal Medical College Hospital, Sirajgonj, Bangladesh.

4. Assistant Professor, Department of Surgery, North Bengal Medical College Hospital, Sirajgonj, Bangladesh.

5. Ex-Prof of Obs \& Gynae, Dhaka Medical College, Dhaka.

6. Medical Officer (MO), Institute of Nuclear Medicine \& Allied Sciences, Bangladesh Atomic Energy Commission, Dinajpur

7. Professor\& HOD, Department of Surgery, KhwajaYunus Ali Medical College Hospital, Sirajgonj. Bangladesh.

Correspondence: M Abdur Rouf, Associate Professor of Surgery, KhwajaYunus Ali Medical College, Enayetpur, Sirajgonj, Bangladesh. E-mail: abdurroufdr@gmail.com Mobile: +8801718910341. 
(Multi System Organ Failure) etc.], Omphalophobia etc. Persistence of the urachus (allantois) or vitelline duct at the umbilicus may cause troubles in early or late life. ${ }^{3-5}$ Other benign and cancerous diseases of the umbilicus include keloids, dermatofibroma, cholesteatoma, malignant melanoma, primary umbilical malignancy and umbilical metastasis (secondary the well-known Sister Mary Joseph nodule). ${ }^{6-8}$ Omphalolith (also known as umbolith, omphalith, omphalo-keratolith, umbilical bolus, inspissated umbilical bolus) is not a common condition under normal circumstances. Howbeit, in case of a deeply retracted umbilicus in unhygienic persons, irrespective of age and sex, especially in obese individuals, the accumulation of dirts, dead skin cells, sebum and keratin may lead to the formation of multiple small concretions which gradually can coalesce leading to the development of obvious stones called the navel stones or calculi. ${ }^{9-11}$ On oxidation specially in contact with air, these calculi may take a blackish hue. ${ }^{12}$ These calculi may remain undetected for several years until and unless complicated by ulceration or infection or hemorrhage etc. ${ }^{2,13}$

\section{Case Report}

A 20-year old adult, non-diabetic non-hypertensive non-alcoholic poor male farmer hailing from Sirajgonj visited at the outpatient department. He used to live in a poorly hygienic environment. His personal life and habit were not ideally hygienic. He presented with a purulent discharge from the umbilicus for one year. It was associated with throbbing pain, intermittent fever of mild and moderate gradepreceded by chills and rigors for fifteen days. No other symptoms like nausea, vomiting and discharge of feces, blood or urine from the umbilicus were reported. He gave no past history of notable disease and surgery. He was treated with various types of antibiotics, analgesics, antipyretics for a long time by many physicians of his locality. But the condition was not improved. The patient failed to mention the name of all drugs he took. Examination of the umbilicus showed an active purulent discharge with inflamed, edematous, red skin around the umbilicus, local riseof temperature and tenderness. A palpable mass was present measuring about $2 \times 1.5 \mathrm{~cm}$ just below and adherent to the umbilicus. The mass was hard in consistency. It was irreducible, non-pulsatile, non-compressible and not trans illuminating (Figure: 1). There were no visible and no palpable impulse on coughing. The purulent discharge was sent to the laboratory for culture and sensitivity test, Gram's staining, AFB staining and other studies.

Under spinal anaesthesia, the patient was placed in the supine position. The abdomen was cleaned and draped. After further cleaning the umbilicus, a probe was introduced through the umbilicus to gauge the depth of the lesion. It had not passed beyond the abdominal wall into the peritoneal cavity indicating that it was a sinus and not a fistula. The probe was negotiated to touch the concretions and was thus helpful to presume the diagnosis. A smile incision was made below umbilicus. The incision was deepened and the flap was raised upwards. Multiple calculi of variable consistency were found at a depth of the umbilicus. These were extracted (Figure: 2 ). The sinus tract and the accompanying small abscess cavity were excised. The abscess cavity was about $5 \mathrm{ml}$ size. The sinus tract and the abscess cavity were sent for histopathological examination, culture and sensitivity test, Gram's staining and other studies. The studies revealed Staphylococcus aureus sensitive to gentamycin and amikacin. Histopathological examination that revealed acute inflammatory cells with no granuloma and no malignancy. The patient was given parental antibiotics as per the sensitivity result plus metronidazole against possible anaerobic co-infection in the post-operative period. The post-operative outcome was smooth and uneventful. The patient remained well and asymptomatic on review over six months.

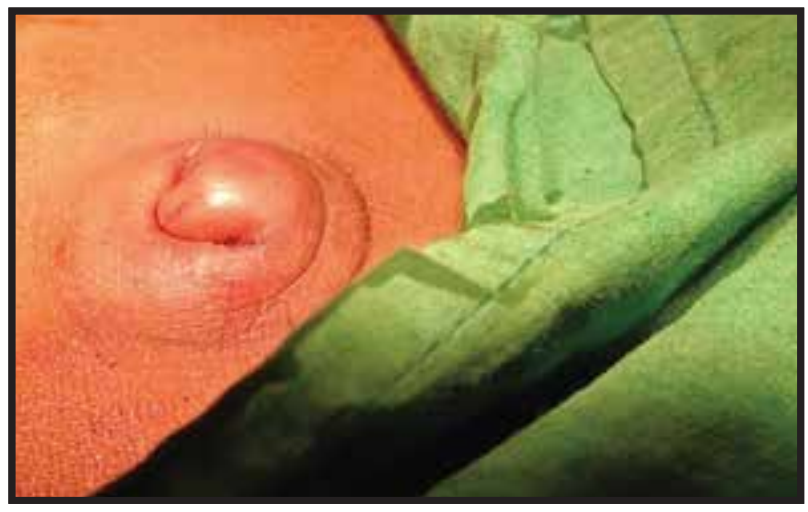

Figure 1: Umbilical sepsis with cellulitis

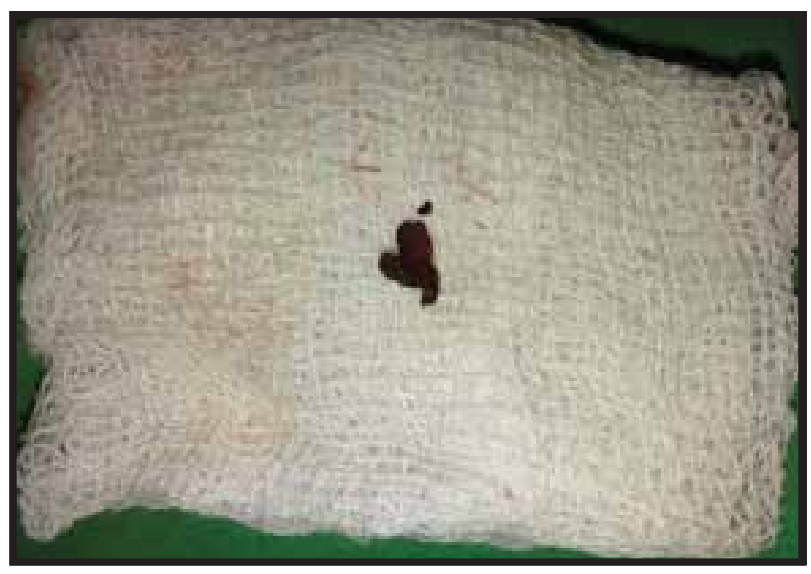

Figure 2: Umbolith

\section{Discussion}

Omphaloliths are very rare finding. ${ }^{1,3}$ They are classically and commonly asymptomatic. Patients very often seek medical advice only if complicated as evidenced by pain, inflammation or infection, discharge or unusual umbilical mass-like suspicious neoplastic lesion. ${ }^{5}$ Poor hygiene is the main cause and an umbilical crevice deep enough to contain significant concretions is a definite prerequisite. When symptomatic, omphalolith commonly presents with a firm to hard, black umbilical mass, resembling a malignant melanoma or sarcoma. Patients may complain of pain and discordant umbilical discharge. It may very often be associated with seborrhea, 
pyogenic infection, peri-umbilical cellulitis, edema, abscess etc. In some countries like Japan, there is a superstition that cleansing of "umbilical sesame" is causative of abdominal pain. ${ }^{7}$ Thus their superstition keeps them unhygienic predisposing to acquired umbilical disorders including umbilical lithiasis. Of course, umbolith is a rare disease. Thence awareness and pre-occupied knowledge are of utmost important for preoperative diagnosis \& to differentiate it from other umbilical disorders. ${ }^{9,13}$

The diagnosis is generally arrived at based on careful history and probing of sinus may feel 'foreign body' sensation during clinical examination though occasionally imaging may be needed like transabdominal ultrasonogram, ${ }^{5}$ MRI and CT scan in early symptomatic or doubtful cases. ${ }^{10,12}$ Rarely, the diagnosis may be arrived at only after surgical exploration of umbilicus. ${ }^{6,7}$

MRI study reveals a well-defined signal void of both T1- and T2-weighted sequences with no contrast enhancement. ${ }^{11}$ Upon preoperative definite diagnosis, the treatment of choice is dilating the umbilical orifice (external opening of the sinus) by the surgeon to extract the calculi and to cleanse the umbilicus with or without excision of sinus tract etc. ${ }^{6,7}$ If infection persists, one is to do umbilical excision (umbilectomy) to remove the resistant infected tissue. ${ }^{2}$

To prevent the calculi first from appearing and to prevent the recurrence, adequate hygiene is to be forced. In exceptional cases, umbilectomy (umbilical excision) is to be done to prevent recurrence. 9,10

\section{Conclusion}

Umbilicus is an important essential anatomical landmark for different surgical procedures. It is also regarded as a beauty part for fashionable women. Excision of umbilicus is a definite treatment option of umbilical sepsis. However, umbilicus may be preserved with proper treatment and elimination of predisposing factors. Preoperative diagnosis is to be done by awareness and pre-occupied knowledge of the attending physicians and surgeons. Though umbolith is a rare disease, it can kill a patient inviting infection and sepsis when complicated and neglected.

\section{Acknowledgement}

The authors express their heartfelt thanks to the patient for allowing the use of his case history and images for publication on academic interest.

\section{References}

1. Pawde SA, Chougale AD, Kulkarni RM, Kadiyal AM, Gowtham T, Kulkarni SS. Umbolith with umbilical abscess and omphalitis: a rare case.Int Surg J. 2020;7(3):898-899.

2. Nittala PP. Omphalolith/umbolith: CT and MR imaging appearances in two patients. Singapore Med J. 2009;50(7):745-746

3. Kumar SKL, Reddy CO, Reddy K. Omphalolith. Indian J Surg. 2011; 73(3): 238-239.

4. RodriguesG.Umbolith: a cause of umbilical discharge and omphalitis. Infection. 2015; 43:625.

5. Salati SA. Omphalolith: A case report. J. Pak. Assoc.Dermatol. 2014; 24 (2):183-185.

6. Burns T, Breathnach S, Cox N, Griffiths C.The general, perineal and umbilical regions. Rook's text book of dermatology, volume 4, 7th edn. Blackwell Science, 2004; $68: 102-168$

7. Mahdi HR, El Hennawy HM. Omphalolith presented with peritonitis: a case report. Cases J. 2009;2(1):8191.

8. Sheehan D, Hussain S, Vijayaraghavan G. Umbilical concretion. J Radiol Case Rep. 2011;5(4):25.

9. Salim G, Touafiq H, Afaf A, ZahraMF. Omphalolith: a rare entitybut important to recognize.Dermatol. Online J. 2014; 20(5): 14 .

10. de Silva WD, Samarasinghe MC, Dias MN, Perera CS. Ectopic gastric and pancreatic tissue: a rare cause of umbilical discharge. Ann Trop Paediatr. 2010;30(1):73-75.

11. Hijran RM and Hany ME. Omphalolith presented with peritonitis: a case report. Cases J. 2009, 2:8191.

12. Nix TE, Young CJ.Congenital umbilical anomalies. Arch Dermatol .1964; 90:160-165.

13. Russel RCG, Williams NS, Bulstrode CJK (eds).Bailey and love's short practice of surgery, 24th edn. Holder Arnold, London, (2004): 1288. 